\title{
Rapid target selection of object categories based on verbs: Implications for language-categorization interactions
}

\author{
Geertje van Bergen $^{1,2}$ | Monique Flecken ${ }^{1,2}$ | Rachel $\mathrm{Wu}^{3}$ (D)
}

\author{
${ }^{1}$ Max Planck Institute for \\ Psycholinguistics, Radboud University \\ Nijmegen, Nijmegen, The Netherlands \\ ${ }^{2}$ Donders Institute for Brain, Cognition and \\ Behaviour, Radboud University Nijmegen, \\ Nijmegen, The Netherlands \\ ${ }^{3}$ Department of Psychology, University of \\ California, Riverside, Riverside, California
}

\section{Correspondence}

Rachel Wu, Department of Psychology, University of California, Riverside, 900 University Avenue, Riverside, CA 92521.

Email: Rachel.Wu@ucr.edu

Funding information

Netherlands Organisation for Scientific

Research (NOW) Veni grant (275-89-022)

(to G.v.B.)

\begin{abstract}
Although much is known about how nouns facilitate object categorization, very little is known about how verbs (e.g., posture verbs such as stand or lie) facilitate object categorization. Native Dutch speakers are a unique population to investigate this issue with because the configurational categories distinguished by staan (to stand) and liggen (to lie) are inherent in everyday Dutch language. Using an ERP component $(\mathrm{N} 2 \mathrm{pc})$, four experiments demonstrate that selection of posture verb categories is rapid (between 220-320 ms). The effect was attenuated, though present, when removing the perceptual distinction between categories. A similar attenuated effect was obtained in native English speakers, where the category distinction is less familiar, and when category labels were implicit for native Dutch speakers. Our results are among the first to demonstrate that category search based on verbs can be rapid, although extensive linguistic experience and explicit labels may not be necessary to facilitate categorization in this case.
\end{abstract}

\section{KE Y W O R S}

categorization, labels, N2pc

\section{1 | INTRODUCTION}

Noun labels facilitate processing and grouping objects with similar perceptual features, such as cars with a similar shape (Connell, 2018; Gentner \& Simms, 2011; Lupyan \& Lewis, 2017; Plunkett et al., 2008; Wu, Mareschal, \& Rakison, 2011). By triggering a mental representation of a canonical representation (i.e., prototype) of a particular category based on perceptual features, labels can "jump-start" visual processes (Boutonnet \& Lupyan, 2015). The majority of prior research on the role of language in object perception and categorization has focused on noun labels. Although verbs have been shown to facilitate learning how one object can relate to another (e.g., a cat chasing a mouse; Gentner, 2006), it is not clear how verbs might facilitate object categorization. An important difference between noun and verb labels is that the former may allow the formation of precise mental representations of canonical category members (e.g., a canonical cat), although broad, higher-level noun categories (e.g., food) do not afford such precise mental representations. By contrast, verbs typically describe relational information, such as objects placed on their natural base relative to a ground object (i.e., standing) for posture verbs. Thus, they evoke less precise mental representations of a category, as multiple verbs can apply to the same item in different instances and multiple objects can apply to the same verb.

Yet, within a familiar language context, it is clear whether an object belongs in a particular verb category (e.g., whether an object is standing or lying for native Dutch speakers). Native Dutch speakers, in contrast to native English speakers, are a unique population with which to investigate object categorization based on posture verbs. The distinction between staan (to stand) versus liggen (to lie) is highly familiar to native Dutch speakers, as it is ingrained in the language used 
for everyday speech (Ameka \& Levinson, 2007; Lemmens, 2002): posture verbs are obligatory to describe the relation between a figure and a ground (i.e., an object on a surface). For example, de sleutels liggen op tafel directly translates to "the keys lie on the table." By contrast, in English, object configurations are typically described with the verb to be, rather than a posture verb, such as "the keys are on the table." The distinction between staan and liggen can be perceptual in nature, where standing objects are often taller than they are wide (a standing bottle), and lying objects are wider than they are tall (a lying bottle). However, this is not the case in all instances. For example, a standing plate is wider than it is tall, whereas a lying ball is as tall as it is wide. The distinction in Dutch posture verbs is based on properties of the object itself: if the object has a natural base, then staan can be used; if not, then only liggen is applicable. In addition, the specific configuration of the object in relation to the ground is relevant for the distinction: for objects with a natural base (a bottle or a plate), staan is used when the object is resting on its base, whereas liggen is used in other cases. Dutch posture verbs thus do not simply encode the horizontal versus vertical orientation of a single object; rather, they mark more complex object configurations.

To investigate the robustness of verb category representations, the present study used a category visual search task. Category search is a fundamental, everyday task, ranging from searching for your keys in different orientations (i.e., a specific category of visual percepts) to searching for something to eat (i.e., a broad category of objects). Because top-down (i.e., goal-directed) visual search requires a mental representation of the target (e.g., keeping in mind what your keys look like when searching for your keys), category-based visual search paradigms provide information about the effects of categorization due to stronger or weaker mental representations of a category. EEG measures, particularly the N2pc ERP component, provide information on the timing of these processes. The N2pc is the earliest and most reliable marker of target selection, emerging approximately $200 \mathrm{~ms}$ after stimulus onset (Eimer, 1996; Luck \& Hillyard, 1994). Prior N2pc category search studies have shown that categories based on nouns (e.g., letters, numbers, faces, kitchen items; Nako, Wu, \& Eimer, 2014; Nako, Wu, Smith, \& Eimer, 2014; Wu et al., 2015) and even adjectives (e.g., healthy vs. unhealthy food; Wu, Pruitt, Zinszer, \& Cheung, 2017) can elicit reliable N2pc components. These studies have demonstrated that searching for categories, especially highly familiar noun categories, can elicit N2pc components that can be as large as, and can emerge as quickly as, searching for a specific item, which affords a clear mental representation. When the mental representation of a category is less precise because not all items in the category share similar perceptual features, such as with healthy versus unhealthy foods, the N2pc component, although still present, becomes attenuated (e.g., Nako, Wu, Smith, et al., 2014; Wu, McGee, Echiverri, \& Zinszer, 2018; Wu, Pruitt, Runkle, Scerif, \& Aslin, 2016).

The present study investigated the mental representations of categories based on posture verbs: to stand versus to lie. If such verb labels evoke mental representations similar to those of familiar noun labels, this finding would suggest that verb labels impact categorization and subsequent attentional processes related to the categories. Although perceptual categories elicit clear mental representations based on prototypes, demonstrating a robust $\mathrm{N} 2 \mathrm{pc}$ component even after removing the perceptual nature of the distinction would provide stronger evidence that verb labels can impact mental representations of categories, extending previous work on the role of language (nouns) on visual attention. By reducing prior linguistic experience with the categories (via testing non-Dutch speakers, i.e., native English speakers) or by investigating differences in effects between explicit versus implicit category labels, we can study in more detail the contribution of verb labels on categorization.

The present study demonstrates that searching for categories based on the verbs staan versus liggen can elicit N2pc components in native Dutch speakers when the categories are labeled explicitly and when the objects conform to the prototypical perceptual distinction (standing $=$ taller, lying = wider; Experiments $1 \mathrm{~A}$ and $1 \mathrm{~B}$ ). In addition, a reliable but attenuated N2pc was elicited even when removing the perceptual distinction for the explicitly labeled categories: standing and lying categories now both contained mostly wider than taller objects (Experiment 2). An attenuated N2pc effect also was found in English speakers when the categories were explicit (Experiment 3), demonstrating that there may not be an obvious benefit for category search in this task related to extensive prior linguistic experience with the verb categories. Finally, an attenuated but present N2pc was elicited in Dutch speakers when the categories were implicit (i.e., not explicitly labeled), suggesting that, at least in familiar language contexts, explicit verb labels may not be necessary in this task to elicit the category effects obtained (Experiment 4).

\section{$2 \mid$ EXPERIMENT 1}

\section{1 | Method}

Experiments 1A and 1B used the same or different objects between categories to investigate whether categories based on posture verbs would elicit an N2pc during category search when the objects conformed to canonical representations of the categories (i.e., standing $=$ tall, lying $=$ wide). 


\subsection{1 | Participants}

Following prior N2pc category search studies (e.g., Wu et al., 2016), data from 16 native Dutch speakers (nine female, $M=22.8$ years, $S D=2.61$, range $=18-28$ ) were included in the final analyses for Experiment 1A. An additional six participants were excluded from the final analyses due to excessive eye movements ( $>50 \%$ of trials excluded due to horizontal eye movements). Experiment $1 \mathrm{~B}$ included another sample of 16 Dutch native speakers (10 female, $M=21.4$ years, $S D=2.28$, range $=18-27$ ). Data from an additional three participants were excluded from the analyses of Experiment 1B due to excessive eye movements. Participants in both experiments were tested at the Max Planck Institute for Psycholinguistics and provided informed consent. All had normal or corrected-to-normal vision and were paid $€ 18$ at the end of the experiment.

\subsection{2 | Stimuli}

The stimuli, in part adapted from van Bergen and Flecken (2017), were created using Blender, an open-source animation software (www.blender.org). The stimuli consisted of grayscale images of household objects placed on a table (eight objects for Experiment 1A, and 16 objects for Experiment 1B). For Experiment 1A, each object had two configurations between categories: one configuration was standing (Figure 1 , upper), and the other was lying (lower). Using identical objects across categories ensured that each object from the standing category was visually identical (except for its orientation) to its counterpart in the lying category. In Experiment 1B, each object occurred in only one configuration, belonging to either the standing or the lying category (Figure 2). Most stimuli conformed to canonical representations of the categories (standing $=$ tall, lying $=$ wide). The images subtended $3.6^{\circ} \times 3.6^{\circ}$ and were presented at $3.03^{\circ}$ from the central fixation point on a 24 -inch $60 \mathrm{~Hz}$ Dell monitor.

\subsection{3 | Design and procedure}

Both experiments included two category search tasks: one that involved searching for any standing object and another for any lying object. In addition, an exemplar search task was included to confirm that the individual objects can elicit N2pc components (e.g., search for a specific object in a specific configuration; see online supporting information, Appendix S1). The task order for category and exemplar search was varied across participants. For example, one participant may have completed the tasks in the following order: exemplar search, standing category search, lying category search, whereas another may have completed the following order: lying category search, exemplar search, standing category search. The same stimuli were displayed in all conditions
Experiment $1 \mathrm{~A}$
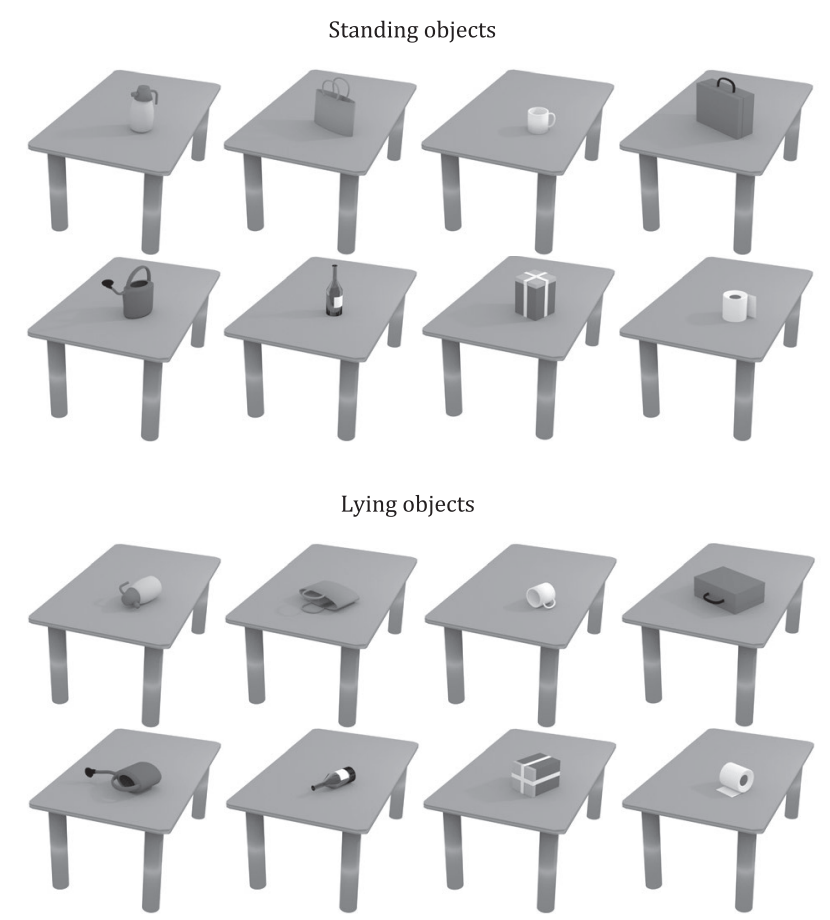

F IG URE 1 Standing (upper) and lying (lower) objects used as search stimuli in Experiment $1 \mathrm{~A}$

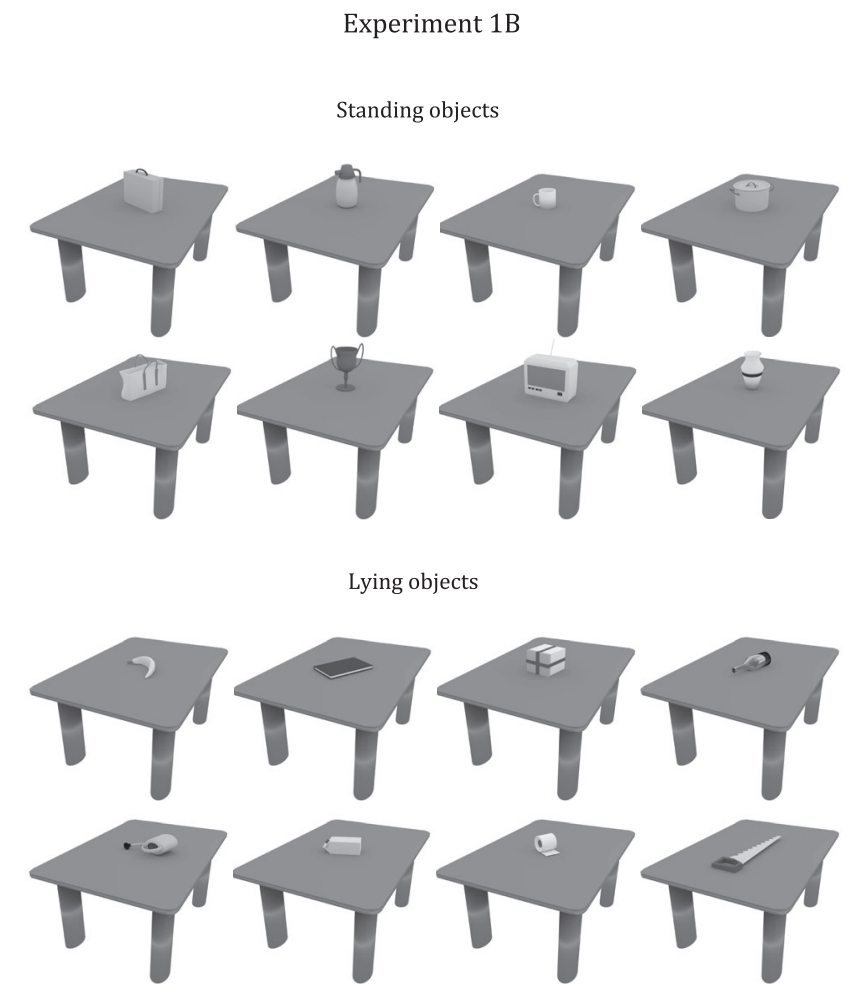

F I G URE 2 Standing and lying objects used as search stimuli in Experiment 1B 
throughout the study for all participants. Therefore, only the instructions determined whether particular stimuli were the targets or distractors.

The category search tasks each included eight blocks, and each task included 224 category match trials and 224 no category match target trials. Category match trials consisted of any object from the target category appearing on one side, while an object from the no target category appeared on the other side, and no category match trials displayed only objects from the nontarget category. Each of the eight blocks contained 28 category match trials and 28 no category match trials. At the beginning of the experimental session, participants were provided with a printout with all of the objects presented simultaneously under their category label to help them complete the task. Participants were allowed to study the printouts as long as they wanted to, typically not more than a few minutes. The experimenter pointed to which item or items were the target on the printouts before each task. In addition, for the exemplar search tasks, participants were shown the exact target image in the center of the screen before the start of each block until they advanced to the search trials. For the category search tasks, participants were shown a black icon of a standing or a lying person before each block to indicate search for a standing or lying category, respectively. Participants searched for the same target for all eight blocks.
The search array presented two objects simultaneously on the left and right side of a black fixation dot on a white background for $200 \mathrm{~ms}$. This array was followed by a $1,600-\mathrm{ms}$ interstimulus interval displaying only the white background and the fixation dot (Figure 3). As a constraint in N2pc studies, participants fixated the dot throughout the experiment and searched for targets via their peripheral vision. Participants also were instructed to press the right (green) button on a button box with their right index finger when a target was present and the left (red) button with their left index finger when the target was absent. A target present response was required on half of the trials and a target absent response on the other half. Participants had $1,800 \mathrm{~ms}$ to respond to the array from stimulus onset until the next array, and they were asked to respond as quickly and as accurately as possible. This design was similar to that from $\mathrm{Wu}$ and colleagues (Wu, McGee, Echiverri, et al., 2018; Wu, McGee, Rubenstein, et al., 2018; Wu et al., 2016, 2017).

\subsection{4 | EEG recording, data preprocessing, and analyses}

The EEG data were recorded from 27 cap-mounted active electrodes placed according to the 10-20 system and four active electrodes placed at the outer canthi of both eyes and above and below the center of the left eye to capture blinks and horizontal eye movements. Electrode impedances were kept below $20 \mathrm{k} \Omega$.

\section{Sample trial sequence}

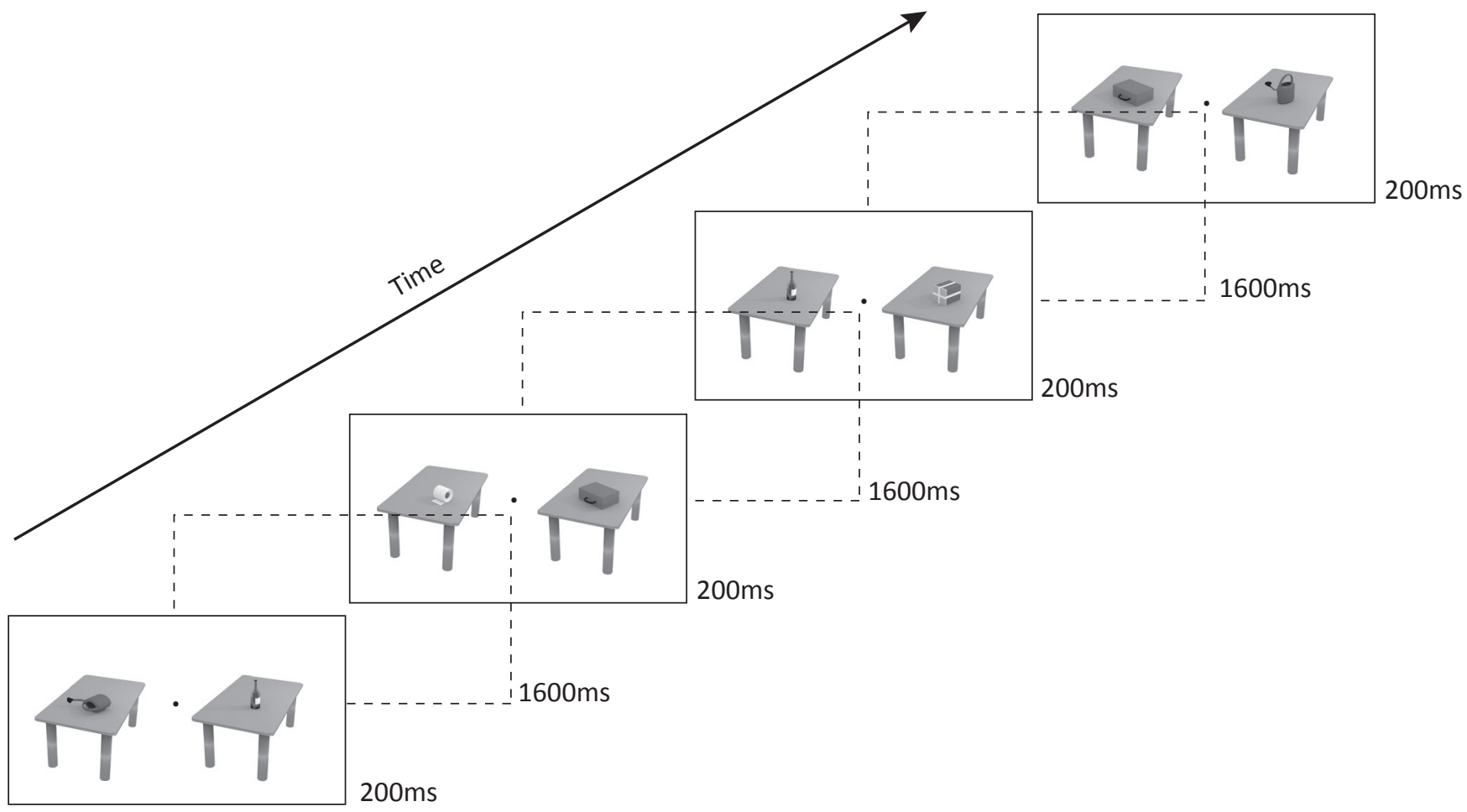

F I G U RE 3 Example search arrays and trial sequence from the search tasks in all experiments 
Signals were amplified using BrainAmp DC amplifiers with a band-pass filter between 0.01 and $150 \mathrm{~Hz}$ and digitized at a sampling frequency of $500 \mathrm{~Hz}$. Data were recorded in reference to the left mastoid and rereferenced offline to the average of the two mastoids. Bipolar vertical (VEOG) and horizontal (HEOG) electro-oculogram signals were calculated from the right and left VEOG and HEOG channels.

We used a 100-ms prestimulus baseline period for epochs from $-100 \mathrm{~ms}$ to $500 \mathrm{~ms}$ relative to the search array onset. For the artifact rejection criteria, we used HEOG exceeding $\pm 25 \mu \mathrm{V}$, VEOG exceeding $\pm 60 \mu \mathrm{V}$, all other channels exceeding $\pm 80 \mu \mathrm{V}$. For all ERP analyses, only trials with a correct response from $100 \mathrm{~ms}$ to $1,500 \mathrm{~ms}$ after stimulus onset were included. We retained $79 \%$ of all correct trials on average per participant after rejecting trials based on eye movement artifacts. We used the 220-320 ms time window after search array onset to assess the mean N2pc amplitude at lateral posterior electrodes PO7 and PO8 (cf. Wu et al., 2015).

\section{$2.2 \quad$ Results}

\subsection{1 | ERP}

Only category match trials (rather than no category match trials) were included in the ERP analyses because the contralateral nature of the $\mathrm{N} 2 \mathrm{pc}$ component requires identifying the location of a target in the analyses. The ERP results of all experiments are illustrated in Figures 4 and 5, and the mean $\mathrm{N} 2 \mathrm{pc}$ amplitudes are listed in Table 1. A paired samples $t$ test between contralateral and ipsilateral mean amplitudes revealed a significant $\mathrm{N} 2 \mathrm{pc}$ component for category match trials: Experiment $1 \mathrm{~A}-t(15)=5.98, p<0.001$, Experiment $1 \mathrm{~B}-t(15)=4.98, p<0.001$. Comparing the N2pc components between Experiments $1 \mathrm{~A}$ and $1 \mathrm{~B}$, an independent samples $t$ test on the difference waves did not reveal a significant difference, $t(30)=1.06, p=0.30$. The lack of a difference between the N2pc components in Experiments 1A and $1 \mathrm{~B}$ suggests that increased category complexity via additional objects did not affect attentional target selection in our paradigm. These results suggest that participants rapidly selected category targets possibly based on posture verb labels, although the obvious perceptual distinction (i.e., standing $=$ tall, lying $=$ wide) may have played a role .

\subsection{2 | Behavioral}

We compared participants' accuracy and (log-transformed) reaction times between category match trials and no category match trials separately for each experiment (Figure 6, Table 1). For Experiment 1A, accuracy for category match trials was significantly lower than that for no category match trials, $t(15)=-2.59, p=0.02$. Reaction times for category match trials were significantly faster for no category match trials, $t(15)=-4.46, p<0.01$. For Experiment 1B, accuracy did not significantly differ between category match trials and no category match trials, $t(15)=0.98, p=0.34$, but reaction times for category match trials were faster than that for no category match trials, $t(15)=-4.53, p<0.01$. These results suggest that correctly selecting a category target may be largely similar in difficulty as correctly indicating that no target was present.

To investigate whether there were differences between experiments, we conducted two 2 (Trial Type) $\times 2$ (Experiment) analyses of variance (ANOVAs) - one for accuracy and one for reaction time. Results from the accuracy analysis revealed no significant main effect of experiment, $F(1,30)=1.89, p=0.18$, nor a significant interaction, $F(1,30)=0.77, p=0.39$. Similarly, for reaction times, we found no evidence for a main effect of experiment, $F(1,30)=1.03, p=0.32$, nor evidence for an interaction, $F(1,30)=0.93, p=0.34$. These results suggest that searching for a more varied set of objects may not be too impactful on behavioral outcomes during category search based on posture verb labels.

\section{$3 \mid$ EXPERIMENT 2}

\section{1 | Method}

Given that the N2pc is highly affected by perceptual salience and pop-out effects, to evaluate the influence of verb concepts on the N2pc and therefore categorization, we minimized the perceptual distinction between the two categories. In this experiment, objects from both categories were similarly wide and tall. Therefore, category membership was now determined only by the concepts provided by the verbs, rather than by perceptual distinctions.

\subsection{1 | Participants}

Sixteen Dutch native speakers (14 female, $M=22.1$ years, $S D=3.97$, range $=18-30)$ participated in Experiment 2. Participants were tested at the Max Planck Institute for Psycholinguistics. The data from an additional six participants were excluded from the final analyses due to excessive eye movements. All participants had normal or corrected-tonormal vision and were compensated $€ 18$.

\subsection{2 | Stimuli, design, and procedure}

The stimuli consisted of 16 household objects placed on a table, eight in each category (Figure 7). The objects in both categories tended to be wider than they were tall or similar in height and width (e.g., a mug), and each object from the standing category was visually similar to an object from the lying category (e.g., a standing printer vs. a lying suitcase). The design and procedure were identical to those from Experiments $1 \mathrm{~A}$ and $1 \mathrm{~B}$, as were EEG recording and data 



F I G U RE 4 Grand-averaged ERPs elicited by search arrays for category match trials at posterior electrodes PO7/8 contralateral and ipsilateral to a category target in all experiments. N2pc difference waveforms were obtained by subtracting ipsilateral from contralateral ERP waveforms

preprocessing procedures. We retained $77 \%$ of all correct trials on average per participant after rejecting trials based on eye movement artifacts.

\section{2 $\quad$ Results}

\subsection{1 | ERP}

A paired samples $t$ test between contralateral and ipsilateral mean amplitudes revealed a significant $\mathrm{N} 2 \mathrm{pc}$ component for category match trials, $t(15)=4.68, p<0.001$. An independent samples $t$ test comparing the N2pc in Experiment 1B and
Experiment 2 revealed that the $\mathrm{N} 2 \mathrm{pc}$ was significantly smaller in Experiment 2 compared to Experiment $1 \mathrm{~B}, t(30)=2.75, p$ $=0.01$. These data suggest that verb labels may facilitate categorization and category search, although the effect is attenuated (though still present) when perceptual cues are removed.

\subsection{2 | Behavioral}

We found no difference between category match trials and no category match trials in accuracy, $t(15)=-1.80, p=$ 0.09 , but reaction times were significantly faster for category match trials than for no category match trials, $t(15)=-9.10$, 


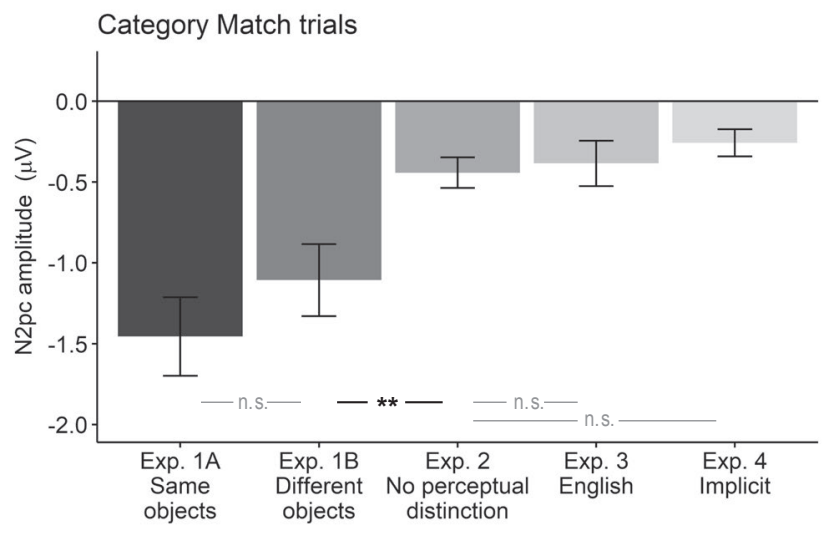

F I G U RE 5 Mean N2pc amplitudes for category match trials from all experiments. A one-way ANOVA across experiments and follow-up $t$ tests revealed that Experiments 1A and 1B did not differ, and both were larger than Experiments 2, 3, and 4, which did not differ. Error bars represent $\pm 1 S E$

$p<0.001$ (Figure 6). An omnibus analysis showed that accuracy was similar across Experiments $1 \mathrm{~B}$ and 2 (all $F \mathrm{~s}<1$ ). An omnibus analysis of reaction times revealed a significant main effect of experiment, $F(1,30)=11.06, p<0.01$, but no significant interaction, $F(1,30)=0.09, p=0.77$, showing that reaction times were slower in Experiment 2 compared to those from Experiment $1 \mathrm{~B}$ for both category match and no category match trials. These results demonstrate that, when removing perceptual cues from a category search task, the task becomes more difficult.

\section{EXPERIMENT 3}

\section{1 | Method}

Experiment 3, with native English speakers, investigated whether the N2pc elicited from the first two experiments were due to the Dutch participants' extensive experience with the verb categories. If linguistic experience does not play a crucial role, then we would find a similarly large N2pc between Experiments 2 and 3. To evaluate the explicit influence of verb concepts on categorization in native English speakers, we used the stimuli from Experiment 2, which minimized the perceptual distinctions between the two categories.

\subsection{1 | Participants}

Data from 16 English native speakers (11 female, $M=22.2$ years, $S D=4.68$, range $=18-35$ ) were analyzed. Six additional participants were excluded from the final analyses due to excessive eye movements. Participants were tested at Brain and Cognitive Sciences at the University of Rochester. All had normal or corrected-to-normal vision and were paid $\$ 25$ at the end of the experiment.

\subsection{2 | Stimuli, design, and procedure}

The stimuli were identical to those from Experiment 2, as were the design and procedure. Instead of a button box, participants responded on a keyboard with right and left arrow keys. The EEG data were DC-recorded at standard positions of the extended 10-20 system $(500 \mathrm{~Hz}$ sampling rate, $40 \mathrm{~Hz}$ low-pass filter) using 32 active electrodes. The EEG data were rereferenced offline to the averaged earlobes. Data preprocessing and analyses were identical to the procedures from Experiments 1 and 2. We retained $72 \%$ of all correct trials on average per participant after eye-movement artifact rejection.

\section{$4.2 \quad$ Results}

\subsection{1 | ERP}

A paired samples $t$ test between contralateral and ipsilateral mean amplitudes revealed a significant $\mathrm{N} 2 \mathrm{pc}$ component for category match trials, $t(15)=2.74, p=0.02$
TAB L E 1 Mean and $S D$ values for EEG and behavioral measures

\begin{tabular}{|c|c|c|c|c|c|c|c|}
\hline & & \multicolumn{2}{|c|}{$\mathbf{N} 2 p c(\mu V)$} & \multicolumn{2}{|c|}{ Accuracy } & \multicolumn{2}{|c|}{ RT (ms) } \\
\hline & & $M$ & $S D$ & $M$ & $S D$ & $M$ & $S D$ \\
\hline \multirow[t]{5}{*}{ Category match } & Experiment $1 \mathrm{~A}$ & -1.45 & 0.97 & 0.92 & 0.10 & 572 & 74 \\
\hline & Experiment 1B & -1.11 & 0.88 & 0.90 & 0.08 & 589 & 71 \\
\hline & Experiment 2 & -0.44 & 0.38 & 0.86 & 0.09 & 676 & 80 \\
\hline & Experiment 3 & -0.38 & 0.56 & 0.89 & 0.05 & 684 & 71 \\
\hline & Experiment 4 & -0.26 & 0.34 & 0.89 & 0.04 & 683 & 63 \\
\hline \multirow[t]{5}{*}{ No category match } & Experiment 1A & & & 0.96 & 0.04 & 608 & 76 \\
\hline & Experiment $1 \mathrm{~B}$ & & & 0.92 & 0.05 & 638 & 71 \\
\hline & Experiment 2 & & & 0.90 & 0.09 & 737 & 98 \\
\hline & Experiment 3 & & & 0.94 & 0.04 & 748 & 83 \\
\hline & Experiment 4 & & & 0.90 & 0.09 & 750 & 72 \\
\hline
\end{tabular}

Abbreviation: RT, reaction time. 

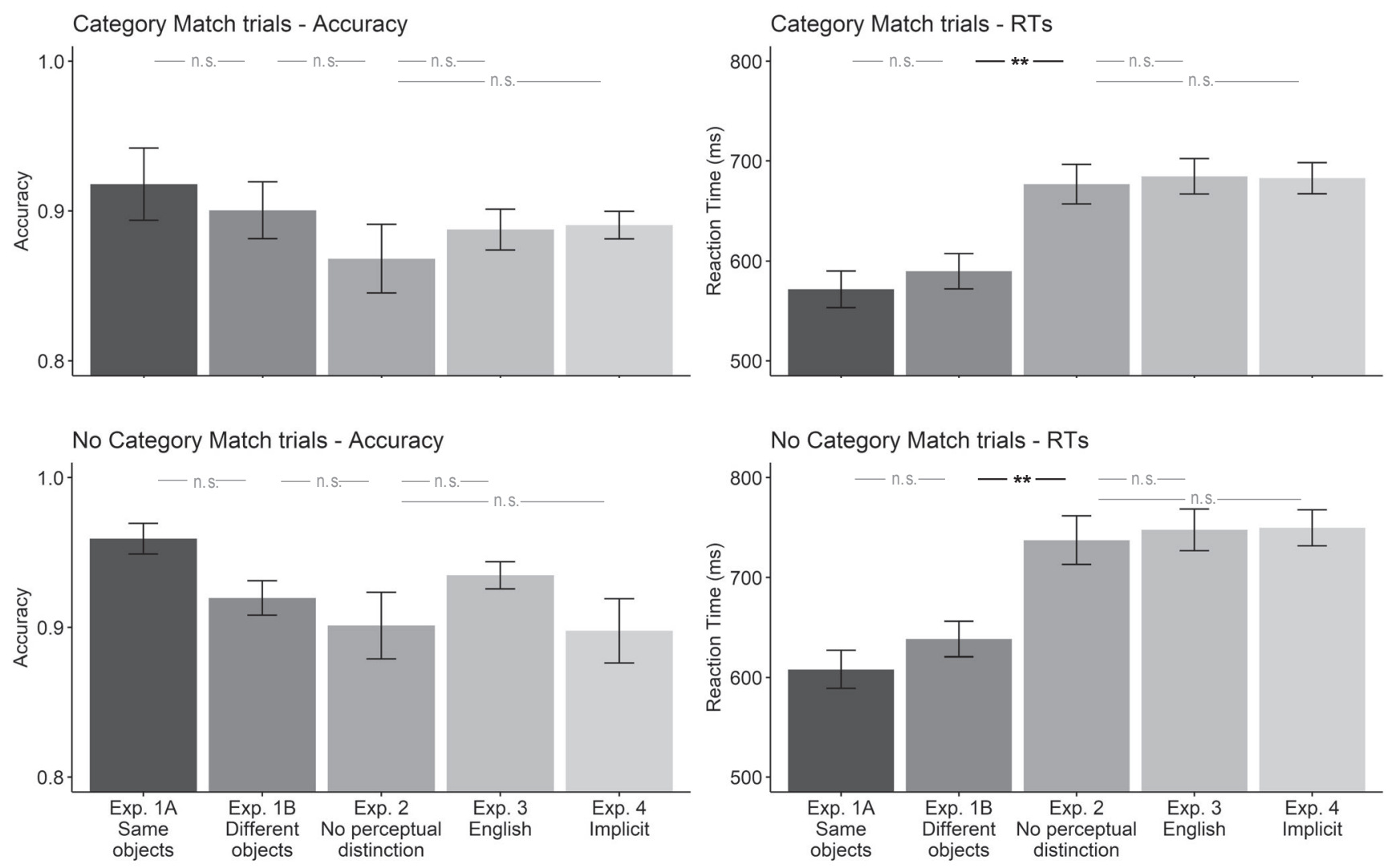

F I G URE 6 Accuracy (left) and response times (right) for category match trials (upper) and no category match trials (lower) from all experiments. Error bars represent $\pm 1 S E$

Experiment 2

Standing objects

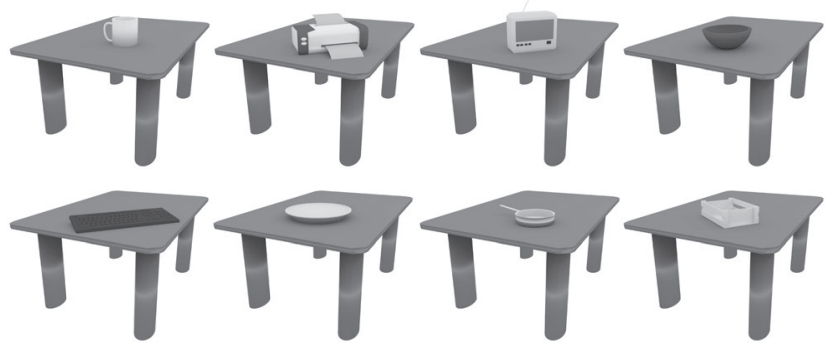

Lying objects

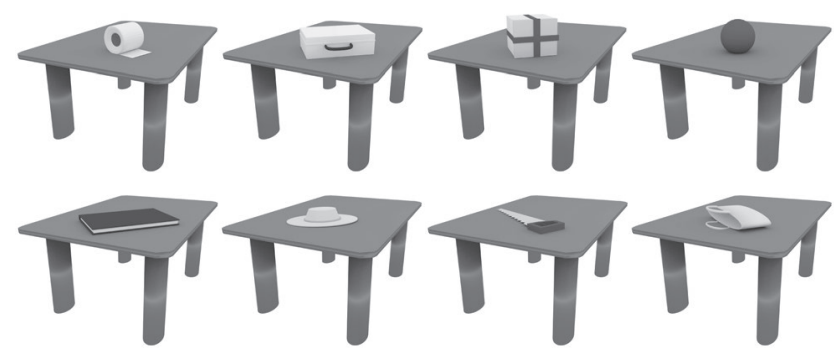

F I G U RE 7 Standing and lying objects used as search stimuli in Experiments 2, 3, and 4
(Figures 4 and 5). In other words, we found an N2pc when native English speakers searched for the relatively less familiar verb categories. Comparing the N2pc for category match trials between Experiment 2 (Dutch-speaking participants) and Experiment 3 (English-speaking participants), a $t$ test revealed no significant difference, $t(30)$ $=-0.34, p=0.73$, suggesting that familiarity based on extensive linguistic experience may not play an impactful role on search in this case.

\subsection{2 | Behavioral}

Category match trials and no category match trials differed significantly in accuracy, $t(15)=-4.2, p<0.01$, and reaction times, $t(15)=-6.41, p<0.01$, such that category match trials elicited faster but less accurate responses than no category match trials (Figure 6). Omnibus analyses showed no significant differences in accuracy or reaction times between Experiments 2 and 3 (all $F \mathrm{~s}<1.3, p \mathrm{~s}>0.25$ ). These results suggest that native English speakers with lower prior exposure to the verb categories were able to complete the task similarly to native Dutch speakers with a lifetime of experience.

These findings suggest that linguistic experience may not be as relevant for this task (similar to other studies 
demonstrating little to no effect of prior experience on category search tasks; Wu et al., 2015; Wu et al., 2017). However, studies have found effects on exemplar trials due to prior experience (see supporting information, Appendix S1; e.g., Wu et al., 2017, Wu, McGee, Echiverri, et al., 2018; Wu, McGee, Rubenstein, et al., 2018). Although a lifetime of experience with the linguistic categories may not impact category search, it may be the case that the English participants quickly learned the categories after they were labeled explicitly. To investigate whether category search for configurational categories with no clear perceptual distinction can still be successful without explicit labels, Experiment 4 included Dutch participants who were presented with the categories from Experiment 2, but without explicit verb labels (i.e., the categories were referenced only as Category A and Category B). In prior research, implicit linguistic distinctions have been shown to facilitate perceptual discrimination and categorization (e.g., color, space; Holmes \& Regier, 2017; Malt \& Majid, 2013; Regier \& Kay, 2009). If labels played a substantial role in Experiment 2 with Dutch participants (for whom the distinction is familiar) and if the labels were quickly learned and applied in Experiment 3 with English participants, we would expect to see a smaller N2pc effect, if any, in Experiment 4.

\section{5 | EXPERIMENT 4}

\section{1 | Method}

To evaluate the implicit influence of verb concepts on categorization, we used the stimuli from Experiment 2, which minimized the perceptual distinctions between the two categories, without providing an explicit label for the standing and lying categories.

\subsection{1 | Participants}

We analyzed data from 16 Dutch native speakers (12 female, $M=22.1$ years, $S D=3.97$, range $=18-30$ ). Two additional participants were excluded from the final analyses due to excessive eye movements. All participants were tested at the Max Planck Institute for Psycholinguistics, had normal or corrected-to-normal vision, and were paid $€ 18$.

\subsection{2 | Stimuli, design, and procedure}

All aspects were identical to those from Experiment 2, except that the categories were not explicitly labeled as standing or lying, but rather labeled as Category A and Category B. Therefore, the prompts presented at the beginning of each block to indicate the target category were capitalized, boldfaced letters A and B instead of standing or lying icons of a person. Per participant, we retained $78 \%$ of all correct trials on average after eye-movement artifact rejection.

\section{$5.2 \mid$ Results}

\section{\begin{tabular}{l|l} 
5.2.1 & ERP
\end{tabular}}

A $t$ test revealed a significant $\mathrm{N} 2 \mathrm{pc}$ component for category match trials, $t(15)=3.05, p=0.01$ (Figures 4 and 5). Comparing the N2pc on category match trials between Experiment 2 (explicit) and Experiment 4 (implicit) revealed no significant difference, $t(30)=-1.47, p=0.15$, suggesting that explicit labels may not be necessary to elicit a category N2pc in our task, at least in familiar language contexts.

\subsection{2 | Behavioral}

Category match trials and no category match trials did not differ significantly in accuracy, $t(15)=-0.39, p=0.70$, but responses to category match trials were faster than responses to no category match trials, $t(15)=-8.41, p<0.01$ (Figure 6). Omnibus analyses showed that accuracy and reaction times were similar across both Experiments 2 and 4 (all $F$ s $<1$ ).

When asking participants at the end of the experimental session what strategy they used to remember which object belonged to the categories, not a single participant mentioned the objects' orientation. These results suggest that explicit labels do not provide additional benefits to category search in familiar language contexts in this task.

\section{6 | DISCUSSION}

Four experiments used ERPs and behavioral measures to investigate category target selection based on posture verbs (e.g., search for any standing object) in native Dutch speakers (familiar context) and native English speakers (unfamiliar context). In Experiment 1A and Experiment 1B, when Dutch participants were allowed to rely on canonical object configurations associated with the verbs (i.e., standing = tall, lying $=$ wide), target selection was rapid, within 220$320 \mathrm{~ms}$. This time window is similar to that for searching for a specific object (e.g., a standing vase; Nako, Wu, Smith, et al., 2014). Experiment 2 reduced Dutch participants' ability to rely on such perceptual features by including only objects that were wider than they were tall or similar in width and height in both categories. Results from Experiment 2 revealed an $\mathrm{N} 2 \mathrm{pc}$ in the same time window, although the effect was attenuated compared to the N2pc from Experiment 1B.

Two subsequent experiments investigated the role of language experience (native Dutch speakers vs. native English speakers; Experiment 3) and explicit versus implicit labels for Dutch speakers (Experiment 4). Due to the nature of the English language, English speakers have less experience categorizing objects based on posture verbs. Results from Experiment 3 revealed an attenuated but reliable N2pc for 
English speakers, similar to the N2pc for Dutch speakers from Experiment 2 (using identical stimuli and tasks). Experiment 4 investigated with Dutch speakers the impact of explicit versus implicit category verb labels. When labels were implicit, we found a small but reliable N2pc that was similar to the N2pc from Experiment 2 (where labels were explicit).

The finding that verb categories based on object configurations can lead to efficient category search is noteworthy (i.e., a reliable $\mathrm{N} 2 \mathrm{pc}$ in all of the experiments), albeit the effects are stronger with perceptual distinctions between categories. Thus far, N2pc studies have demonstrated that only categories distinguished by object types via noun or adjective labels (rather than object relations via verb labels) can be selected by approximately $200 \mathrm{~ms}$ to $220 \mathrm{~ms}$ (e.g., letters, numbers, faces, kitchen items: Nako, Wu, \& Eimer, 2014; Nako, Wu, Smith, et al., 2014; Wu et al., 2015; healthy vs. unhealthy food: Wu et al., 2017). The results from the present studies are among the first to show that information from configurations can be useful for categorization and category search.

The results from Experiments $2-4$ allow us to better understand the role of verb labels for categorization and category search, although some questions still remain. In Experiment 2, perceptual cues were minimized, resulting in object configurations that do not conform to the canonical perceptual features associated with the verbs. The reliable N2pc in Experiment 2 may indicate that verb labels facilitate category search, even with less canonical object configurations. The presence of an N2pc in Experiment 3, then, would suggest that English participants had quickly learned the categories after they were labeled explicitly. However, prior studies have shown that even advanced English learners of Dutch (in contrast with native Dutch speakers) do not use the configurational information encoded in posture verbs to guide their visual attention to potential sentence continuations in incremental language comprehension (van Bergen \& Flecken, 2017). Moreover, the category N2pc in Experiment 4 would imply that the linguistic categories in Dutch participants are so deeply entrenched that they elicit an N2pc even in the absence of an explicit label. However, none of the participants in Experiment 4 reported using object configuration as a strategy for categorization during the task, suggesting that even the strategy itself was implicit or nonexistent. Therefore, it remains unclear how participants completed the task in Experiments 2-4, and it may be the case that participants used different strategies to complete the task in each experiment.

It may be possible that participants performed a hybrid search task, using both visual and memory search (e.g., Wolfe, 2012) without categorizing the items. Hybrid search is typically characterized by a linear increase in search times as the number of items in a search array increases and a logarithmic increase as the number of items in memory sets increases. Given that all memory and search array set sizes were held constant throughout all four experiments, any differences we found across experiments were likely not due to hybrid search per se. Our findings are more in line with Cunningham and Wolfe (2014), who suggest that potential targets are compared in parallel to items from categories in long-term memory, although the timing may depend on the nature of the category (e.g., the specificity of the items within the category). Moreover, we have shown in prior studies that eight objects in a memory set that are not grouped into a category do not elicit an N2pc during category search, even if reaction times do not differ much between items that are grouped versus not grouped (e.g., Wu et al., 2016; Wu, McGee, Echiverri, \& Zinszer, 2018). We therefore take the presence of a reliable $\mathrm{N} 2 \mathrm{pc}$ in the present experiments as evidence that the participants performed the task categorically.

Future studies could further investigate the role of verbs on category search by comparing search based on familiar categories and search based on unfamiliar ad hoc categories, such as having different lying and standing objects in both categories. However, unfamiliar ad hoc categories are difficult for participants to learn in one experimental setting, especially when it is not clear how the objects are connected (e.g., Wu et al., 2013). Therefore, comparing search for an unfamiliar ad hoc category to search for a familiar verb category might overestimate the contribution of verbs on category search. Perhaps comparing search for noun versus verb categories would be informative, but such a comparison is beyond the scope of the present studies, which investigated the role of verbs on category search.

It is possible that showing the participants all of the objects within their respective categories prior to starting the experiment may have helped them complete the task. However, to maximize the amount of clean data during the experimental session, we aimed to minimize online learning by familiarizing the participants to the objects beforehand. Future studies where participants perform a category search without prior familiarization to the stimuli would be informative, even if the data are noisier. A study including novel images on every trial would contribute to determining the robustness of the category effect. In such studies, thousands of objects per category are required for one experimental session. A study at this scale indeed would be informative and is now justified, given our positive findings in the present studies with a limited stimulus set.

Another possible explanation for our finding that Experiments 1A and 1B had larger N2pc components than Experiment 2 is that verb labels activate typical category members, rather than atypical category members, a view advocated by the label-feedback hypothesis (Lupyan \& Thompson-Schill, 2012). Labels are especially useful cues for perceptual processing, precisely because their function is to generalize across 
individual exemplars and activate a prototypical representation of a percept. Under this interpretation, the posture verbs accompanying the categories in Experiments $1 \mathrm{~A}$ and $1 \mathrm{~B}$ highlighted the canonical configuration distinction between standing and lying, and therefore may have enhanced $\mathrm{N} 2 \mathrm{pc}$ components relative to Experiments 2-4. The similar N2pc effects in Experiments 2 and 3 (native Dutch speakers vs. native English speakers unfamiliar with the linguistic categories in Dutch), as well as in Dutch participants without explicit verb labels (Experiment 4), suggest that categorization of atypical exemplars (object configurations without a clear perceptual distinction) on the basis of posture verbs does not afford as strong of a mental category representation compared to categorization of typical exemplars. Therefore, it is possible that the difference between the N2pc between Experiments 1A, 1B and Experiments 2-4 may have been partially driven by canonical items in the natural category, which conform to the perceptual cues.

The present study adds to a growing literature demonstrating the value and constraints of category representations on visual search. Although further empirical work is needed to clarify the role of verb labels on category search, our findings suggest the potential value, as well as limits, of verbs when performing a fundamental, everyday task.

\section{ACKNOWLEDGMENTS}

We thank Gary Lupyan and Richard Aslin for providing helpful feedback on these studies, which were partly funded by the Netherlands Organisation for Scientific Research (NWO, Veni grant 275-89-022, awarded to G. van Bergen).

\section{ORCID}

\section{Rachel Wu (D) https://orcid.org/0000-0002-7486-697X}

\section{REFERENCES}

Ameka, F. K., \& Levinson, S. C. (Eds.). (2007). The typology and semantics of locative predication: Posturals, positionals and other beasts [Special issue]. Linguistics, 45.

Boutonnet, B., \& Lupyan, G. (2015). Words jump-start vision: A label advantage in object recognition. Journal of Neuroscience, 24, 93299335. https://doi.org/10.1523/jneurosci.5111-14.2015

Connell, L. (2018). What have labels ever done for us? The linguistic shortcut in conceptual processing. Language, Cognition and Neuroscience. Advanced online publication. https://doi. org/10.1080/23273798.2018.1471512

Cunningham, C. A., \& Wolfe, J. M. (2014). The role of object categories in hybrid visual and memory search. Journal of Experimental Psychology: General, 143(4), 1585-1599. https://doi.org/10.1037/ a0036313

Eimer, M. (1996). The N2pc component as an indicator of attentional selectivity. Electroencephalography and Clinical Neurophysiology, 99, 225-234. https://doi.org/10.1016/0013-4694(96)95711-9
Gentner, D. (2006). Why verbs are hard to learn. In K. Hirsh-Pasek \& R. Golinkoff (Eds.), Action meets word: How children learn verbs (pp. 544-564). Oxford, UK: Oxford University Press.

Gentner, D., \& Simms, N. (2011). Language and analogy in conceptual change. Behavioral and Brain Sciences, 34, 128-129. https://doi. org/10.1017/S0140525X10002736

Holmes, K. J., \& Regier, T. (2017). Categorical perception beyond the basic level: The case of warm and cool colors. Cognitive Science, 41, 1135-1147. https://doi.org/10.1111/cogs.12393

Lemmens, M. (2002). The semantic network of Dutch posture verbs. In J. Newman (Ed.), The linguistics of sitting, standing and lying (pp. 103-140). Amsterdam, The Netherlands: John Benjamins.

Luck, S. J., \& Hillyard, S. A. (1994). Electrophysiological correlates of feature analysis during visual search. Psychophysiology, 31, 291308. https://doi.org/10.1111/j.1469-8986.1994.tb02218.x

Lupyan, G., \& Lewis, M. (2017). From words-as-mappings to wordsas-cues: The role of language in semantic knowledge. Language, Cognition and Neuroscience. Advanced online publication. https:// doi.org/10.1080/23273798.2017.1404114

Lupyan, G., \& Thompson-Schill, S. L. (2012). The evocative power of words: Activation of concepts by verbal and nonverbal means. Journal of Experimental Psychology: General, 141, 170-186. https ://doi.org/10.1037/a0024904

Malt, B. C., \& Majid, A. (2013). How thought is mapped into words. Wiley Interdisciplinary Reviews: Cognitive Science, 4, 583-597. https://doi.org/10.1002/wcs.1251

Nako, R., Wu, R., \& Eimer, M. (2014). Rapid guidance of visual search by object categories. Journal of Experimental Psychology: Human Perception and Performance, 40, 50-60. https://doi.org/10.1037/ a0033228

Nako, R., Wu, R., Smith, T. J., \& Eimer, M. (2014). Item and category-based attentional control during search for real-world objects: Can you find the pants among the pans? Journal of Experimental Psychology: Human Perception and Performance, 40, 1283-1288. https://doi.org/10.1037/a0036885

Plunkett, K., Hu, J.F. \& Cohen, L.B. (2008). Labels can override perceptual categories in early infancy. Cognition, 106, 665-681.

Regier, T., \& Kay, P. (2009). Language, thought, and color: Whorf was half right. Trends in Cognitive Science, 13, 439-446. https://doi. org/10.1016/j.tics.2009.07.001

van Bergen, G., \& Flecken, M. (2017). Putting things in new places: Linguistic experience modulates the predictive power of placement verb semantics. Journal of Memory and Language, 92, 26-42. https ://doi.org/10.1016/j.jml.2016.05.003

Wolfe, J. M. (2012). Saved by a log: How do humans perform hybrid visual and memory search? Psychological Science, 23(7), 698-703. https://doi.org/10.1177/0956797612443968

Wu, R., Mareschal, D., \& Rakison, D. H. (2011). Attention to multiple cues during spontaneous object labeling. Infancy, 16, 545-556. https ://doi.org/10.1111/j.1532-7078.2010.00061.x

Wu, R., McGee, B., Echiverri, C., \& Zinszer, B. D. (2018). Prior knowledge of category size impacts visual search. Psychophysiology, 55, e13075. https://doi.org/10.1111/psyp.13075

Wu, R., McGee, B., Rubenstein, M., Pruitt, Z., Cheung, O. S., \& Aslin, R. N. (2018). Emergence of the benefits and costs of grouping for visual search. Psychophysiology, 55, e13087. https://doi.org/10.1111/ psyp.13087

Wu, R., Nako, R., Band, J., Pizzuto, J., Ghoreishi, Y., Scerif, G., \& Aslin, R. N. (2015). Rapid selection of non-native stimuli despite 
perceptual narrowing. Journal of Cognitive Neuroscience, 27, 2299-2307. https://doi.org/10.1162/jocn_a_00857

Wu, R., Pruitt, Z., Runkle, M., Scerif, G., \& Aslin, R. N. (2016). A neural signature of rapid category-based target selection as a function of intra-item perceptual similarity despite inter-item dissimilarity. Attention, Perception, \& Psychophysics, 78, 749-776. https://doi. org/10.3758/s13414-015-1039-6

Wu, R., Pruitt, Z., Zinszer, B., \& Cheung, O. (2017). Increased experience amplifies the activation of task-irrelevant category representations. Attention, Perception, \& Psychophysics, 79, 522-532. https:// doi.org/10.3758/s13414-016-1254-9

Wu, R., Scerif, G., Aslin, R., Smith, T. J., Nako, R., \& Eimer, M. (2013). Searching for something familiar or novel: Top-down attentional selection of specific items or object categories. Journal of Cognitive Neuroscience, 25, 719-729. https://doi.org/10.1162/jocn_a_00352

\section{SUPPORTING INFORMATION}

Additional supporting information may be found online in the Supporting Information section at the end of the article.

\section{Appendix S1}

How to cite this article: van Bergen G, Flecken M, Wu R. Rapid target selection of object categories based on verbs: Implications for languagecategorization interactions. Psychophysiology. 2019;56:e13395. https://doi.org/10.1111/psyp.13395 